\title{
SUSTITUCIÓN DEL MAÍZ POR HARINA INTEGRAL DE ZAPALLO EN LA NUTRICIÓN DE POLLOS DE ENGORDE
}

\section{REPLACING CORN MEAL BY BUTTERNUT SOUASH FLOUR IN BROILER CHICKENS NUTRITION}

\author{
Cristian Camilo Ubaque ${ }^{1}$, Lucy Viviana Orozco ${ }^{2}$, Sanín Ortiz $^{3}$, Magda Piedad Valdés ${ }^{4}$, Franco Alirio Vallejo ${ }^{5}$
}

\begin{abstract}
${ }^{1}$ Zootecnista. Universidad Nacional de Colombia, Sede Palmira, Colombia, e-mail: ccubaquep@unal.edu.co; ${ }^{2}$ Zootecnista. Universidad Nacional de Colombia, Sede Palmira, Colombia, e-mail: lvorozcoa@unal.edu.co; ${ }^{3}$ Zootecnista, Ph.D. Universidad Nacional de Colombia, Sede Palmira, Colombia, e-mail: sortizg@unal.edu.co; ${ }^{4}$ Ingeniera Agroindustrial, PhD. Universidad Nacional de Colombia, Sede Palmira, Colombia, e-mail: mpvaldesr@unal.edu.co; ${ }^{5}$ Ingeniero Agrónomo, PhD. Universidad Nacional de Colombia, Sede Palmira, Colombia, e-mail: favallejoc@unal.edu.co; Facultad de Ciencias Agropecuarias, Universidad Nacional de Colombia. A.A. 237. Palmira, Valle del Cauca, Colombia.
\end{abstract}

Rev. U.D.C.A Act. \& Div. Cient. 18(2): 137-146, Enero-Diciembre, 2015

\section{RESUMEN}

Un total de 144 pollos de engorde de la línea genética CobbAvian, 48 criados hasta los 42 días de edad fueron distribuidos en un diseño experimental de parcelas divididas comprendidas en cuatro tratamientos con tres repeticiones, cada una de ellas con 12 individuos, 6 de cada sexo, con el objetivo de evaluar el efecto de la sustitución del grano de maíz, en un 50, 75 y $100 \%$ por harina integral de zapallo (HIZ), como fuente alternativa de energía, proteína y pigmentos naturales, en el rendimiento general de las aves, durante las fases de crecimiento y finalización. El resultado más sobresaliente, se obtuvo con la ración sustituida en un $50 \%$, que comparada con las demás inclusiones de harina integral de zapallo, obtuvo el promedio más alto de peso vivo, ganancia de peso, peso en canal, conversión y eficiencia alimenticia. Además, este tratamiento no tuvo diferencias significativas $(p<0.05)$ en su rendimiento en canal, con el tratamiento control y presentó bajos contenidos de grasa abdominal y alto grado de pigmentación de la piel. Se estimó, con base en la regresión de la curva de crecimiento de las aves, que se requieren de 3 a 5 días más de encasetamiento, para alcanzar el peso comercial, para el 50\% de sustitución con HIZ.

Palabras clave: Cucurbita moschata, grasa abdominal, pigmentación de la piel, rendimiento en canal, Unapal Abanico 75 .

\section{SUMMARY}

A total of 144, Cobb Avian 48, genetic line broilers, reared up to 42 days of age were distributed in a split plot experimental design with 4 treatments and 3 replicates, each formed by 12 chickens, 6 of each sex, in order to evaluate the substitution of corn at 0, 50, 75 y 100\% by integral pumpkin flour (HIZ), as an alternative energy, protein and natural pigments source in the performance of the birds during the growing and finishing phases. The best results were obtained with the ration $50 \%$ replacement, which compared with other rations with inclusions of HIZ, got the highest averages in live weight, carcass weight, feed conversion and efficiency, without significant differences $(\mathrm{p}<0.05)$ to control treatment in carcass yield, but high differences in the low abdominal fat content and high grade on skin pigmentation, resulting benefited both aspects with the treatment $50 \%$ HIZ included. It valued to according to the regression of the growth curve of the birds that will have been required only 3 to 5 days more of chick placement to reach the commercial weight.

Key words: Cucurbita moschata, abdominal fat, skin pigmentation, carcass yield, Unapal Abanico 75.

\section{INTRODUCCIÓN}

La nutrición del pollo de engorde se ha visto afectada, porque el maíz, la torta de soya, las grasas de origen animal y vegetal, entre otros insumos para la manufactura de alimentos balanceados para animales (ABA), han incrementado de precio, como resultado de la exploración de nuevas tecnologías, para la generación de biocombustibles, como etanol y biodiesel (Corzo, 2008; Brown, 2006).

El maíz es el cereal más utilizado en la nutrición animal, pues participa entre el 60 a $75 \%$ en las dietas empleadas para este fin (Chaves, 2009); sin embargo, en la actualidad, existe un continuo énfasis en investigaciones para el uso de alimentos alternativos, como fuente energética en la producción animal (Monforte et al. 2002). Desde este punto de vista, se ponen 
a consideración el empleo de los recursos locales existentes $y$, entre los cuales, se encuentra una hortaliza de importancia para Colombia, el zapallo (Cucurbita moschata Duch), por su demanda en el consumo, volumen de producción, calidad nutricional (Vallejo \& Estrada, 2004) y por ser fuente natural de pigmentantes (Ortiz et al. 2008; Ortiz et al. 2013).

El fruto de zapallo, se caracteriza por proveer carotenoides (Tobar et al. 2010), sustancias que, en su mayoría, previenen enfermedades carenciales y oncogénicas, en especial, los carotenoides $\alpha$-caroteno, $\beta$-caroteno y Luteína (Ortiz et al. 2008; Jacobo et al. 2011). A nivel agroindustrial, Saeleaw \& Schleining (2011) resaltan el valor de la harina de esta hortaliza, que radica en su contenido de carbohidratos, fibra y almidones.

Con el objetivo de evaluar el efecto de la sustitución parcial y total del maíz por harina integral de zapallo (HIZ) en la nutrición de pollos de engorde, durante las fases de crecimiento (11 a 21 días) y finalización (22 a 42 días), esta investigación consideró la HIZ de la variedad Unapal Abanico 75 como materia prima, debido a su alto contenido de materia seca en fruto, $26 \%$ en promedio (Baena et al. 2010), comparado con el $8 \%$, como referencia estándar del fruto de zapallo, reportada por la USDA, en 2008.

\section{MATERIALES Y MÉTODOS}

El trabajo de campo, se realizó en el Centro Experimental Mario González Aranda, de la Universidad Nacional de Colombia, Sede Palmira, Valle del Cauca, situado a 03³0'26,8” latitud norte y $76^{\circ} 18^{\prime} 47,6^{\prime \prime}$ longitud oeste y altitud de 998 msnm, temperatura promedio de $24^{\circ} \mathrm{C}$, humedad relativa del $72 \%$ y precipitación pluvial anual de 1000 mm (Valdés et al. 2010).

Fueron evaluados cuatro tratamientos o raciones experimentales en una población de aves conformada por 72 hembras y 72 machos, para un total de 144 individuos, en un periodo que comprendió desde los 11 hasta los 42 días de edad: T0: Ración con $100 \%$ maíz, T1: Ración con $50 \%$ maíz y $50 \%$ HIZ, T2: Ración con 25\% maíz y 75\% HIZ y T3: Ración con 100\% HIZ.

Cada tratamiento fue subdivido en tres repeticiones, cada una de ellas estuvo compuesta por 12 individuos repartidos entre machos y hembras, por igual. En cada repetición, se evaluó el desempeño general de las aves, a través del registro semanal del consumo en gramos (C), conversión alimenticia en gramos (CA) y eficiencia alimenticia en porcentaje (EFA). Al momento del sacrificio de las aves, se valoró el peso vivo final en gramos (PVF), peso de la canal en gramos $(P C)$, rendimiento de la canal en gramos (R), el contenido de grasa abdominal en gramos (GA) y el grado de pigmentación de la piel (GPP) valorado de 1 a 15, en la escala de Roche.

Las raciones alimenticias fueron balanceadas de forma isoproteíca e isocaloríca, para las fases de crecimiento y finalización (Tabla 1), de acuerdo a la composición nutricional esperada de las materias primas utilizadas y los requerimientos nutricionales para pollos de engorde de desempeño superior, según las tablas brasileñas para aves y cerdos (Rostagno, 2005).

Tabla 1. Composición de las raciones experimentales elaboradas para las fases de crecimiento y finalización.

\begin{tabular}{|c|c|c|c|c|c|c|c|c|}
\hline \multirow{2}{*}{$\begin{array}{c}\text { Materia prima } \\
\text { (\%) }\end{array}$} & \multicolumn{4}{|c|}{ Crecimiento } & \multicolumn{4}{|c|}{ Finalización } \\
\hline & T0 & T1 & $\mathrm{T} 2$ & T3 & T0 & T1 & $\mathrm{T} 2$ & T3 \\
\hline Maíz & 54,85 & 28,06 & 14,47 & - & 57,12 & 29,94 & 15,05 & - \\
\hline H. Zapallo & - & 28,06 & 43,46 & 59,07 & - & 29,94 & 45,14 & 62,06 \\
\hline Torta soya & 36.52 & 35,07 & 33,77 & 32,83 & 33,17 & 30,94 & 30,29 & 28,93 \\
\hline Aceite palma & 4,54 & 4,71 & 4,20 & 4,10 & 5,91 & 5,38 & 5,62 & 5,21 \\
\hline Biofos & 1,60 & 1,50 & 1,40 & 1,30 & 1,50 & 1,30 & 1,30 & 1,20 \\
\hline C. calcio & 1,33 & 1,30 & 1,30 & 1,30 & 1,20 & 1,20 & 1,20 & 1,20 \\
\hline Lisina & 0,25 & 0,30 & 0,30 & 0,30 & 0,30 & 0,30 & 0,30 & 0,30 \\
\hline Metionina & 0,36 & 0,40 & 0,40 & 0,40 & 0,30 & 0,40 & 0,40 & 0,40 \\
\hline Treonina & 0,05 & 0,10 & 0,20 & 0,20 & - & 0,10 & 0,20 & 0,20 \\
\hline Sal & 0,20 & 0,20 & 0,20 & 0,20 & 0,20 & 0,20 & 0,20 & 0,20 \\
\hline Premezcla & 0,30 & 0,30 & 0,30 & 0,30 & 0,30 & 0,30 & 0,30 & 0,30 \\
\hline Total & 100,00 & 100,00 & 100,00 & 100,00 & 100,00 & 100,00 & 100,00 & 100,00 \\
\hline
\end{tabular}


La cantidad de alimento, se ofreció de acuerdo a lo estipulado en las tablas de consumo existentes para la línea y fue suministrada en dos raciones por día; de igual modo, se realizó el cálculo de CA, con base en el método mostrado en las guías de manejo Cobb (Cobb-Vantress, 2009).

La HIZ, se obtuvo con el protocolo de Ortiz et al. (2008), empleando frutos con pericarpio de $C$. moschata, variedad Unapal Abanico 75, a los cuales, se adicionó una solución antioxidante, compuesta por $1 \%$ sorbato de potasio $\left(\mathrm{C}_{6} \mathrm{H}_{7} \mathrm{KO}_{2}\right), 1 \%$ eritorbato de sodio $\left(\mathrm{C}_{6} \mathrm{H}_{7} \mathrm{NaO}_{6}\right)$ y $1 \%$ benzoato de sodio $\left(\mathrm{NaC}_{6} \mathrm{H}_{5} \mathrm{CO}_{2}\right)$; posteriormente, el producto obtenido fue protegido de la luz, para preservar la calidad del material empleado.

Cumplidos los 42 días de engorde de los animales, se efectuó su sacrificio, empleando tres individuos por sexo y por repetición, para un total de 72 aves valoradas, siguiendo el protocolo descrito por López \& Casp (2004).

Los análisis de laboratorio realizados a la HIZ y las dietas experimentales, se llevaron a cabo en las instalaciones de los laboratorios de Semillas y Química de Suelos, de la Universidad Nacional de Colombia, Sede Palmira y en el laboratorio de Análisis Químico y Bromatológico, de la Universidad Nacional de Colombia, Sede Medellín. Los análisis y sus respectivas metodologías fueron: a) materia seca, mediante AOAC, 1990; b) fibra detergente neutra (FDN) y fibra detergente ácida (FDA), bajo el protocolo de Van Soest; c) proteína bruta basada en NTC 4657; d) energía bruta por calorimetría, basada en ISO 9831 y, e) carotenos totales, según RodríguezAmaya (1997). Adicionalmente, a la harina integral de zapallo (HIZ), se le realizaron las pruebas de: a) fósforo y boro, bajo digestión ácida por técnica de espectroscopia UV-Vis; b) carbono orgánico, por el método de titulación de Walkley \& Black y, c) bases y elementos menores, con la técnica de potenciometría volumen/volumen o peso/volumen.

Para el análisis de las variables productivas de consumo, de conversión alimenticia y de eficiencia alimenticia, se utilizó un diseño de bloques completamente al azar, ya que el suministro de las raciones fue mixto y, por lo tanto, la influencia del sexo no fue medida. El modelo para este caso fue:

$$
Y_{i j}=\mu+R_{i}+T_{j}+E_{i j}
$$

Donde: $Y_{\mathrm{ij}}$ : Variable dependiente, Ri: Repeticiones (Bloques), Tj: Tratamientos, Eij: Error

Para el análisis de los datos obtenidos post-sacrificio, se aplicó un diseño experimental de parcelas divididas, donde las variables dependientes se encontraban influenciadas por tres fuentes de variación: los tratamientos, las repeticiones y el sexo. El modelo planteado fue el siguiente:

$$
\begin{gathered}
Y_{i j k}=\mu+R_{i}+T_{j}+\left(R_{i} * T_{j}\right) i j+S_{k}+(T * S)_{j k}+\left[(R * S)_{i k}+\right. \\
\left.(R * T * S)_{j k k}\right]
\end{gathered}
$$

Donde: $Y_{\mathrm{ijk}}$ Variable dependiente, Ri: Repeticiones (Bloques), $T_{j}$ : Tratamientos (Parcela Principal), Sk: Género (Subparcela)

El análisis estadístico fue realizado utilizando como herramienta el software SAS 9.0.

\section{RESULTADOS Y DISCUSIÓN}

Al comparar la composición nutricional de la HIZ con la reportada por Rostagno (2005) para el grano de maíz (GM) (Tabla 2), destacan, en la materia prima alternativa (HIZ), altos contenidos de macrominerales: calcio, magnesio, potasio y sodio; mayores contenidos de proteína $(47,7 \%>$ GM) y energía $(5,9 \%>G M)$. Estas cifras consolidan el potencial que tiene en calidad nutricional la HIZ, al compararse con la del GM, con excepción del nivel de fibra, donde los valores presentados para Fibra Detergente Neutra (FDN) son similares para ambas materias primas y la Fibra Detergente Ácida (FDA), significativamente menor en el GM, siendo $3,54 \%$ contra $11,0 \%$ del HIZ, variable que se podría considerar como limitante para el uso de esta materia prima de forma integral en monogástricos, porque la digestibilidad de los materiales nutritivos está condicionada por el bajo contenido de las fibras descritas (Durán, 2004).

Emplear el zapallo con pericarpio pretende lograr el aprovechamiento del fruto entero y facilitar su uso en campo, dado que, retirar la cáscara del fruto es un proceso que requiere de tiempo, mano de obra y genera residuos y, por tanto, manejos adicionales para ellos.

El boro, por su parte, es considerado como un mineral traza y se sabe de su importancia en las plantas; sin embargo, no es bien conocida su función como micro-nutriente en los animales, por lo cual, no se encuentra ampliamente referenciado su requerimiento para las aves de corral; no obstante, Underwood \& Suttle (2003) reportan que el boro es un elemento bien tolerado, necesitándose más de $300 \mathrm{mg} / \mathrm{kg}$ MS para que se manifiesten los primeros signos de intoxicación en aves. De acuerdo a lo resultados de los análisis de laboratorio, en la tabla 2, realizados para la HIZ, el contenido de Boro es de $3,5 \mathrm{mg} / \mathrm{kg}$, lo cual, no representa un riesgo de intoxicación para las aves.

Los resultados arrojados por los análisis bromatológicos realizados a las dietas experimentales, a modo de verificación (Tabla 3), muestran la composición nutricional real de las raciones empleadas. Al comparar los aportes energético y proteico esperados, con los suministrados por los análisis, los resultados registrados en la tabla 3 informan un excedente para ambos componentes nutricionales de cada fase, 
Tabla 2. Composición nutricional comparativa entre la HIZ y el maíz.

\begin{tabular}{|c|c|c|c|c|}
\hline \multirow{2}{*}{\multicolumn{2}{|c|}{ Ítem }} & \multirow{3}{*}{$\begin{array}{c}\begin{array}{c}\text { Unidad } \\
\text { HIZ }\end{array} \\
\% \%\end{array}$} & \multicolumn{2}{|c|}{ Valor } \\
\hline & & & \multirow{2}{*}{$\begin{array}{r}\text { Maíz }{ }^{* *} \\
87,76\end{array}$} & \multirow[b]{2}{*}{87,11} \\
\hline MS & Materia seca & & & \\
\hline EB & Energía bruta & $\mathrm{Kcal} / \mathrm{kg}$ & 4157 & 3925 \\
\hline $\mathrm{EM} *$ & Energía metabolizable & $\mathrm{Kcal} / \mathrm{kg}$ & $3402 *$ & 3381 \\
\hline PB & Proteína bruta & $\%$ & 12,20 & 8,26 \\
\hline FDN & Fibra detergente neutra & $\%$ & 12,00 & 11,75 \\
\hline FDA & Fibra detergente ácida & $\%$ & 11,00 & 3,54 \\
\hline $\mathrm{Ca}$ & Calcio & $\%$ & 0,72 & 0,03 \\
\hline $\mathrm{Mg}$ & Magnesio & $\%$ & 1,46 & 0,09 \\
\hline $\mathrm{K}$ & Potasio & $\%$ & 3,55 & 0,28 \\
\hline $\mathrm{Na}$ & Sodio & $\%$ & 0,79 & 0,02 \\
\hline $\mathrm{Fe}$ & Hierro & ppm & 3,53 & 43,10 \\
\hline $\mathrm{Cu}$ & Cobre & ppm & 0,05 & 2,90 \\
\hline $\mathrm{Zn}$ & Zinc & ppm & 0,73 & 19,40 \\
\hline Bo & Boro & ppm & 3,50 & - \\
\hline
\end{tabular}

Dónde: * La Energía Metabolizable (EM) fue estimada mediante la ecuación descrita por Francesch, 2001: EMAn $(\mathrm{Kcal} / \mathrm{Kg} \mathrm{MS})=0,9362$ EB - 15,38 PC - 25,16 Pared celular. ** Información extraída de Rostagno, 2005.

respecto a los requerimientos de las aves: hasta $867 \mathrm{Kcal} / \mathrm{kg}$ de EM y $1,7 \%$ de PC en Crecimiento; $644 \mathrm{Kcal} / \mathrm{kg}$ de EM y 2,3\% de PC en Finalización.

Zanusso et al. (1999) encontraron que a un contenido mayor a los $3.150 \mathrm{Kcal} / \mathrm{kg}$ de EM en las fases de inicio y crecimiento, se comienza a registrar una disminución del rendimiento, siendo la energía el principal factor regulador de la ingesta; el valor calórico de las dietas por encima del requerido, se convierte en un limitante del consumo y, en menor medida, lo hace un exceso en el nivel de proteína (Vaca, 2003; Ferket \& Gernat, 2006). Situación que explica, en primera instancia, la reducción del consumo, observado en la tabla 4 y que se extiende a los valores de conversión y de eficiencia alimenticia.

Otros autores, como Zaman et al. (2008), hallaron que el incremento de energía metabolizable $(>3.350 \mathrm{Kcal} / \mathrm{kg})$ en la dieta aumentó la ganancia de peso y la grasa abdominal, entre otros aspectos deseables en la canal, siempre y cuando existan contenidos bajos de proteína, por lo que la relación Energía/Proteína (E/P) juega un papel importante en el rendimiento de las aves (Vaca, 2003).

Sindik et al. (2008) también reportaron un peso corporal más alto en pollos de engorde, que consumieron una dieta con una relación E/P amplia, dado por el menor porcentaje de proteína presente, respecto a aquellos que consumieron raciones con niveles más altos y con relaciones $\mathrm{E} / \mathrm{P}$ más estrechas; sin embargo, los resultados obtenidos en este estudio difieren con las experiencias descritas por Zaman et al. (2008), en los individuos pertenecientes a los tratamientos con inclusión de HIZ, pero de manera más acentuada en aquellos pertenecientes a los tratamientos T2 y T3; de igual manera, tampoco coinciden con lo expuesto por Sindik et al. (2008), pués ambos tratamientos indican las relaciones E/P más amplias $(E / P=167)$ (Tabla 3$)$ y fueron aquellos, quienes de manera general disminuyeron su rendimiento productivo (Tabla 4), razón por la que autores, como Vaca (2003), señala que cuando la energía de la dieta aumenta, se debe aumentar también el contenido de proteína, para mantener la relación energía/proteína adecuada y la de los otros nutrientes, como vitaminas y minerales.

Respecto al contenido de fibra, se registró un aumento proporcional a medida que se elevó el porcentaje de inclusión de HIZ (Tabla 3), donde FDN varió desde 10,3 hasta 12,9, para las raciones de la fase de crecimiento y desde 8,5 a 12,3, para la fase de finalización. Esta tendencia, se enmarca de manera más notoria en la FDA, donde presentó rangos desde 6,0 hasta 10,7 y de 4,5 hasta 9,9, para las respectivas fases. 
Tabla 3. Resultados de los análisis bromatológicos realizados a las raciones experimentales correspondientes a las fases de crecimiento y finalización.

\begin{tabular}{|c|c|r|r|r|r|}
\hline \multirow{2}{*}{ Componente } & \multirow{2}{*}{ Requerimiento } & \multicolumn{5}{|c|}{ Crecimiento } \\
\cline { 3 - 6 } & - & \multicolumn{1}{|c|}{ T0 } & \multicolumn{1}{|c|}{ T1 } & \multicolumn{1}{c|}{ T2 } \\
\hline EB (Kcal/kg) & - & 4531 & 4819 & 4798 & 4783 \\
\hline EM (Kcal/kg) & 3050 & 3619 & 3917 & 3835 & 3802 \\
\hline PB (\%) & 21,14 & 21,3 & 21,80 & 22,70 & 22,80 \\
\hline Relación E/P & 144 & 170 & 180 & 169 & 167 \\
\hline FDN (\%) & - & 10,3 & 11,7 & 12,2 & 12,9 \\
\hline FDA (\%) & - & 6,0 & 7,3 & 8,3 & 10,7 \\
\hline \multirow{2}{*}{ Componente } & Requerimiento & & Finalización & \\
\hline EB (Kcal/kg) & - & 40 & T1 & T2 & T3 \\
\hline EM (Kcal/kg) & 3150 & 3794 & 3702 & 3678 & 3516 \\
\hline PB (\%) & 19,73 & 21,0 & 21,7 & 22,0 & 21,1 \\
\hline Relación E/P & 160 & 181 & 171 & 167 & 167 \\
\hline FDN (\%) & - & 8,5 & 9,9 & 10,2 & 12,3 \\
\hline FDA (\%) & - & 4,5 & 6,7 & 9,1 & 9,9 \\
\hline
\end{tabular}

EM fue estimada mediante la ecuación descrita por Francesch, 2001.

Tabla 4. Comparación estadística entre tratamientos y por sexo para las variables evaluadas en el lote experimental de pollo de engorde.

\begin{tabular}{|c|c|c|c|c|c|c|c|c|}
\hline \multirow{2}{*}{\multicolumn{2}{|c|}{ Variable }} & \multirow{3}{*}{$\begin{array}{c}\text { Unidad } \\
\text { T0 }\end{array}$} & \multicolumn{4}{|c|}{ Tratamiento } & \multicolumn{2}{|c|}{ Género } \\
\hline & & & T1 & T2 & T3 & Machos & Hembras & \\
\hline C & Consumo & & $3994,60^{a}$ & $3614,10^{b}$ & $3451,20^{b c}$ & $3225,50^{c}$ & - & - \\
\hline $\mathrm{CA}$ & $\begin{array}{l}\text { Conversión } \\
\text { Alimenticia }\end{array}$ & $g$ & $1,64^{a}$ & $1,80^{b}$ & $1,90^{b}$ & $1,95^{b}$ & - & - \\
\hline EFA & $\begin{array}{c}\text { Eficiencia } \\
\text { Alimenticia }\end{array}$ & $\%$ & $60,80^{a}$ & $55,47^{b}$ & $52,87^{b}$ & $51,18^{b}$ & - & - \\
\hline PVI & Peso vivo inicial & $g$ & $233^{a}$ & $233,3^{a}$ & $233,7^{a}$ & $223,8^{b}$ & $231,4^{a}$ & $230,6^{a}$ \\
\hline PVF & Peso vivo final & $g$ & $2388,67^{\mathrm{a}}$ & $1919,17^{b}$ & $1723,11^{\text {bc }}$ & $1640,68^{c}$ & $2057,67^{\mathrm{a}}$ & $1778,19^{b}$ \\
\hline GA & $\begin{array}{c}\text { Grasa } \\
\text { Abdominal }\end{array}$ & $g$ & $36,56^{a}$ & $13,22^{b}$ & $6,89^{\mathrm{bc}}$ & $0,44^{c}$ & $15,72^{a}$ & $12,83^{b}$ \\
\hline PC & Peso de la canal & $g$ & $1648,89^{\mathrm{a}}$ & $1324,22^{b}$ & $1115,67^{c}$ & $1068,22^{c}$ & $1382,67^{\mathrm{a}}$ & $1194,83^{b}$ \\
\hline $\mathrm{R}$ & $\begin{array}{c}\text { Rendimiento } \\
\text { de la canal }\end{array}$ & $\%$ & $68,96^{a}$ & $68,97^{a}$ & $64,72^{b}$ & $65,25^{b}$ & $67,00^{a}$ & $66,94^{a}$ \\
\hline GPP & $\begin{array}{c}\text { Grado de } \\
\text { Pigmentación de } \\
\text { la Piel }\end{array}$ & - & $0^{c}$ & $6.39^{b}$ & $6,94^{a b}$ & $7,50^{a}$ & $5,05^{b}$ & $5,36^{a}$ \\
\hline
\end{tabular}

Letras distintas entre promedios en cada fila son significativamente diferentes $(P<0,05)$. 
Los resultados obtenidos del análisis bromatológico correspondientes a las fibras FDN y FDA no pudieron ser comparados con otras fuentes, debido a la escasez de información respecto a los requerimientos de este componente de la fibra en aves; no obstante, se menciona en la literatura, que una de las características principales de los alimentos para aves son una alta densidad energética - proteica y bajo contenido de fibra cruda, esta última con inclusiones máximas del 5\% (Durán, 2004). Los efectos de la fibra, en especial, de FDN y de FDA, son bien conocidos en rumiantes, asociándose la relación de la FDN, con la regulación del consumo y de la FDA, con la digestibilidad y aprovechamiento de los nutrientes de los alimentos (Cobos et al. 2001; Hoffman et al. 2007).

Jorgensen et al. (1996) evaluaron el efecto de diferentes fuentes de fibra en el desarrollo del tracto digestivo y la digestibilidad de los nutrientes en pollos de engorde, concluyendo que el uso de fibra puede disminuir el contenido de materia seca en las heces, aumentar la producción de excretas en relación con el consumo y generar transito rápido, con la consecuente intervención en la absorción de nutrientes.

El consumo acumulado (Tabla 4) fue estadísticamente mayor $(P<0,05)$ en el tratamiento control, seguido por T1 y T2, hasta presentar el menor desempeño en T3; pero al comparar este parámetro en todos los tratamientos, con lo reportado para la línea genética, el consumo mixto acumulado esperado es de 4517g (Cobb-Vantress, 2009), siendo significativamente menor $(\mathrm{P}<0,05)$ en todos los tratamientos, hecho que se pudo manifestar como resultado de la presentación de la dieta.

En este aspecto, numerosos autores concuerdan en que la granulometría de la ración, después de la calidad y composición nutricional de los ingredientes, es el factor con mayor efecto en la función digestiva de los aves, pues funciones básicas, como aprehensión e ingestión voluntaria, se ven afectadas por el tamaño y la textura del alimento, siendo lo más apropiado el suministro en pellets (Oviedo, 2012; Ferket \& Gernat, 2006; Vaca, 2003)

García et al. (2011) han documentado que el suministro de harinas a aves de corral perjudica la motilidad del tracto gastrointestinal y eleva el $\mathrm{pH}$ del contenido digestivo; consecuentemente, al aumentar la motilidad digestiva se reduce el contacto entre nutrientes y enzimas, desmejora el índice de conversión y aumenta la incidencia de procesos entéricos, mientras que, los $\mathrm{pH}$ elevados, reducen la solubilidad y la digestibilidad de los nutrientes.

La conversión alimenticia (Tabla 4) demostró un mejor comportamiento para la dieta control, con un valor de 1,64. El tratamiento con inclusión del $50 \%$ de $\mathrm{HIZ}$ registró una CA de 1,80, que comparada con el 1,77 de la conversión registrada por la línea genética empleada (Cobb-Vantress, 2009), no difiere estadísticamente $(\mathrm{P}<0,05)$, situación que permite deducir que, inclusiones con un máximo de $50 \%$ en las raciones para aves, cumplen con los estándares de la línea.

Los individuos de la dieta control registraron un peso vivo y en canal estadísticamente mayor $(\mathrm{P}<0,05)$, con respecto a los demás tratamientos. El tratamiento T1 no presentó diferencias estadísticamente significativas $(P<0,05)$ en el rendimiento en canal comparado con el control y $63,8 \%$ menos de grasa abdominal, comparado con individuos del T0. Este último resultado, se logra explicar con lo expuesto por Savón (2002) y Escudero \& González (2006), quienes afirman que niveles elevados de fibra, producen una reducción en la absorción de colesterol y de lípidos a nivel intestinal.

El grado de pigmentación en piel fue de 6,39 contra 0, en la escala de Roche, para los tratamiento T1 y T0, resultado que se atribuye al contenido de carotenoides en la HIZ, pese a la inclusión de aceite de palma, un ingrediente conocido por su aporte de carotenos (FAO \& OMS, 2013).

Las pruebas de carotenos totales realizadas revelaron, en las raciones alimenticias, un aporte en base seca de $7,84 \mu \mathrm{g} / \mathrm{g}$, del T0, frente a $127,59 \mu \mathrm{g} / \mathrm{g}$, del T1, para la fase de crecimiento y $2,64 \mu \mathrm{g} / \mathrm{g}$ contra $110,83 \mu \mathrm{g} / \mathrm{g}$, para los mismos tratamientos evaluados en la fase de finalización. Para la HIZ pura, el valor encontrado fue de $139,83 \mu \mathrm{g} / \mathrm{g}$, aporte significativamente alto comparado con lo reportado, incluso, para maíces mejorados: 30 a 38,5 $\mu \mathrm{g} / \mathrm{g}$ (Salinas et al. 2008).

Adicionalmente, considerando los efectos benéficos que tiene para la salud humana el uso de zapallo por su contenido de carotenoides, entre los que destacan la reducción de enfermedades cardiovasculares y cancerígenas (Tobar et al. 2010; Jacobo et al. 2011; Saeleaw \& Schleining, 2011), los resultados obtenidos representarían un valor agregado del producto, pues de acuerdo con investigaciones realizadas, algunos carotenoides, como el $\beta$-caroteno, son fuentes importantes de provitamina A. En humanos, esta deficiencia dietética es un problema que afecta a nivel mundial (Jaeger et al. 2012), por lo que se ha buscado suplementar alimentos con carotenoides (Sánchez et al. 1999) y, en este aspecto, se ha probado que el $\beta$-caroteno logra incrementar el contenido de acetato de retinol en la carne de pollos, alimentados con este carotenoide (King et al. 1995), del cual, la harina de zapallo tiene un alto contenido, registrándose entre 3,1 y $7 \mathrm{mg} / 100 \mathrm{~g}$ (Murkovic et al. 2002).

Basados en los resultados anteriores, el tratamiento T1 fue considerado como el más adecuado para la sustitución del maíz, por ello, se efectuaron las regresiones de la ecuaciones obtenidas a partir de las curvas de crecimiento de machos 
(Figura 1) y hembras (Figura 2), con el fin de conocer cuánto tiempo requieren para alcanzar el peso obtenido por los individuos del tratamiento control; de ésta, se dedujo que los machos alcanzarían este peso a los 47 días, mientras que las hembras lo harían a los 45; sin embargo, estos tiempos adicionales de encasetamiento: 5 y 3 días, para machos y hembras, respectivamente, pudieran ser eliminados, si se cambia la presentación granulométrica de las dietas y se realizan los ajustes requeridos para el adecuado balanceo de las raciones a suministrar.
Del presente trabajo de investigación, se concluye que en la HIZ se destaca el contenido de energía, proteína cruda y macrominerales, lo que consolida su valor biológico, como componente en ABA. Industrialmente, la HIZ obtenida de la variedad Unapal Abanico 75, pese a permitir mayor rendimiento en los procesos agroindustriales por su contenido de MS, requiere de una alta inversión en mano de obra y energía para el desarrollo del proceso, a través de métodos artesanales, aspecto que demanda de mejoras en innovación, que logren su optimización y aumenten la competitividad de esta materia prima.

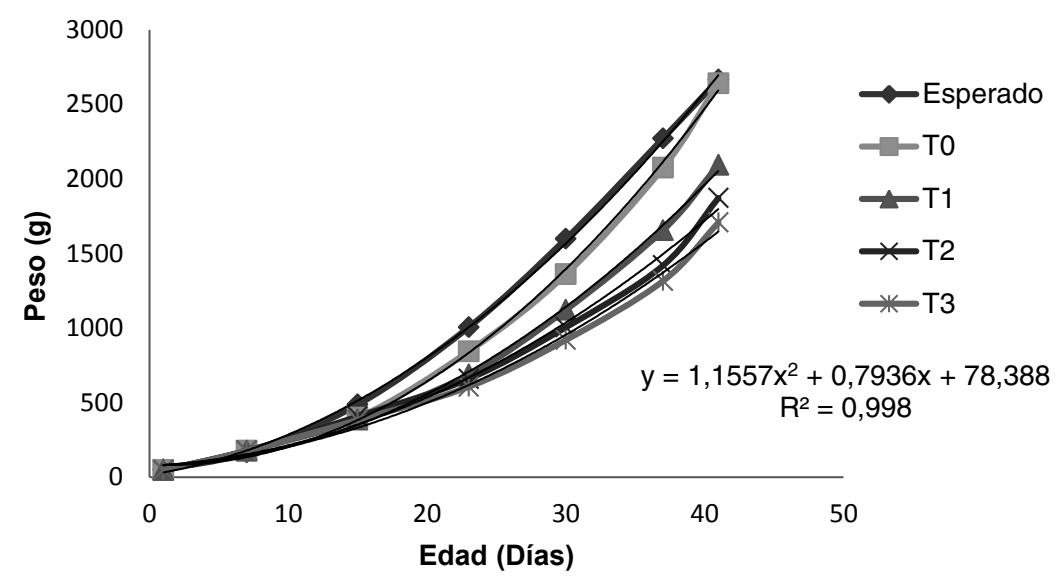

Figura 1. Curva de crecimiento registrada para machos.

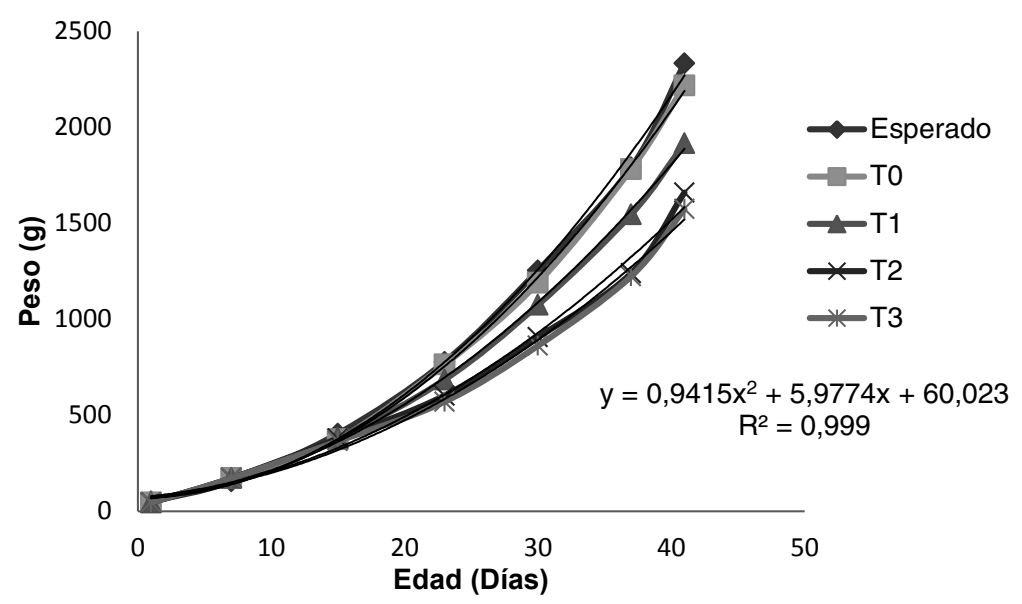

Figura 2. Curva de crecimiento registrada para hembras. 
Agradecimientos: Al grupo de investigación en mejoramiento genético, agronomía y producción de semillas de hortalizas, de la Universidad Nacional de Colombia, sede Palmira, que brindó el apoyo financiero. Conflictos de intereses: El manuscrito fue preparado y revisado con la participación de todos los autores, quienes declaramos que no existe conflicto de intereses que ponga en riesgo la validez de los resultados presentados.

\section{BIBLIOGRAFÍA}

1. BAENA G., D.; ORTIZ G., S.; VALDÉS R., M.P.; ESTRADA S., E.I.; VALLEJO C., .F.A. 2010. UNAPAL - Abanico 75: nuevo cultivar de zapallo con alto contenido de materia seca en el fruto para fines agroindustriales. Acta Agron. 59(3):285292.

2. BROWN, R.L. 2006. La demanda de grano para etanol amenaza la seguridad alimentaria y la estabilidad política. Disponible desde internet en: www.earthpolicy.org (con acceso el 17/10/2013).

3. CHAVES, P.D. 2009. Valoración energética del maíz en dietas de aves. Asociación de Médicos Veterinarios especialistas en Avicultura del Ecuador. Disponible desde internet en: http://www.amevea-ecuador.org/ datos/Valoracion\%20Energetica\%20Maiz\%20en\%20 Dietas\%20de\%20Aves.pdf (con acceso 04/04/2013).

4. COBB - VANTRESS. 2009. Complemento de crecimiento y nutrición del pollo CobbAvian 48. Disponible desde internet en: http://www.cobb-vantress. com/docs/default-source/product-resources/cobbavian48-broiler-performance-and-nutrition-supplement---spanish(grams).pdf?sfvrsn $=0 \quad$ (con acceso 23/09/2012).

5. COBOS, P.M.; SÁNCHEZ, M.; TRINIDAD, S.A.; CETINA, A.V.; VARGAS, H.J. 2001. Importancia del tipo de muestra en la estimación del valor nutritivo de leguminosas y arbustivas, y potencial de un inocuo de bacterias degradadoras de aserrín en sistemas silvopastoriles. En: II Conferencia electrónica de agroforestería para la producción animal en América Latina. Disponible desde internet en: http://www.fao. org/docrep/006/y4435s/y4435s0h.htm (con acceso 11/10/2013).

6. CORZO, A. 2008. Puntos críticos en la nutrición del pollo de engorde. Departamento de Avicultura. Mississippi State University. Disponible desde internet en: http://www.wpsa-aeca.es/aeca_imgs_docs/wpsa1235142257a.pdf (con acceso 10/10/2013).
7. DURÁN, R.F. 2004. Manual de explotación en aves de corral. Grupo Latino Ltda. Bogotá, Colombia. 816 p.

8. ESCUDERO, A.E.; GONZÁLEZ, S.P. 2006. La fibra dietética. Nutr. Hosp. 21(2):61-72.

9. FAO \& OMS. 2013. Revisión de la norma codex stan 210 para aceites vegetales especificados, para la adición del aceite de palma con contenido elevado de ácido oleico (OXG). CX/FO 13/28/8. Comisión del Codex Alimentarius. Disponible desde internet en: ftp://ftp. fao.org/codex/meetings/ccfo/ccfo23/fo23_08s.pdf (con acceso 8/01/2015).

10. FERKET, P.R.; GERNAT, A.G. 2006. Factors that affect feed intake of meat birds: A review. Int. J. Poultry Science. 5(10):905-911.

11. FRANCESCH, M. 2001. Sistemas para la valoración energética de los alimentos en aves. Arch. Latinoam. Prod. Anim. 9(1):35-42.

12. GARCÍA, V.D.; PÉREZ, S.M.; GONZÁLEZ, M.G. 2011. El tamaño de partícula y la presentación del pienso en pollos de engorde. Boletín Selecciones Avícolas. Disponible desde internet en: http://www2.avicultura.com/sa/007-011-Alimentacion-Efecto-particulaGarcia-SA201112.pdf (con acceso 19/02/2013).

13. HOFFMAN, P.C.; LUNDBERG, K.M.; BAUMAN, L.M.; SHAVER, R.D.; CONTRERAS, G.F. 2007. Digestibilidad in vitro del FDN (fibra detergente neutra): el debate de 30 vs 48 horas. Universidad de Wisconsin. Focus on Forage. 5(16):1-4.

14. JACOBO, N.; MAROSTICA, M.R.; ZAZUETA, J.; GALLEGOS, J.A. 2011. Physicochemical, technological properties and health-benefits of Cucurbita moschata Duchense vs. Cehualca: A Review. Food Res. Int. 44(9):2587-2593.

15. JAEGER C., L.M.; BARROS, G.P.; OLIVEIRA G., R.L.; PACHECO, S.; FERNANDEZ M., P.H.; VIANA C., J.L.; REGINI, N.M.; LIMA N., A.C.; RODRIGUES A., V.A.; RAMALHO R., S.R. 2012. Total carotenoid content, $\alpha$ - carotene and $\beta$ - carotene of landrance pumpkins (Cucurbita moschata Duch): a preliminary study. Food Res. Int. 47(2):337-340.

16. JORGENSEN, H.; ZHAO, X.Q.; BACH, K.E.; EGGUM, B.O. 1996. The influence of dietary fibre source and level on the development of the gastrointestinal tract, digestibility and energy metabolism in broiler chickens. British J. Nutr. 75(3):379-395. 
17. KING, A.J.; UIJTTENBOOGAART, T.J.; VRIES, A.W. 1995. $\alpha$ - Tocopherol, $\beta$ - Carotene and ascorbic acid as antioxidants in stored poultry muscle. J. Food Science. 60(5):1009-1012.

18. LÓPEZ, V.R.; CASP, V.A. 2004. Procesos de sacrificio: sacrificio de aves. En: Tecnología de mataderos. Madrid. Mundi-Prensa. p.146-158.

19. MONFORTE, J.; CARIAS, D.; CIOCCIA, A.M.; HEVIA, P. 2002. Valor nutricional de las harinas de Clitoria ternatea y Brachiaria humidicola en la alimentación de pollos de engorde. Interciencia. 27(1):33-38.

20. MURKOVIC, M.; MÜLLEDER, U.; NEUNTEUFL, H. 2002. Carotenoid content in different varieties of pumpkins. J. Food Composit. Analysis 15(6):633-638.

21. ORTIZ, G.S.; BAENA, G.D.; VALLEJO C., F.A.; ESTRADA S., E.I.; VALDÉS R., M.P. 2013. Zapallo para consumo en fresco y fines agroindustriales: investigación y desarrollo. Cali. Feriva. 250p.

22. ORTIZ, G.S.; SÁNCHEZ, L.J.; VALDÉS R., M.P.; BAENA, G.D.; VALLEJO C., F.A. 2008. Retención de caroteno total en fruto de zapallo Cucurbita moschata Duch condicionado por osmodeshidratación y secado. Acta Agron. 57(4):269-274.

23. OVIEDO, R.E. 2012. Factores para mejorar la eficiencia nutricional en pollos de engorda. En: Memorias de la quinta reunión anual de la asociación de especialistas en ciencias avícolas del centro de México A.C. San Juan del Río, Querétaro, México.18-32 p. Disponible desde internet en: http://www.aecacem.mx/ memorias2012.pdf (con acceso 19/02/2013).

24. RODRÍGUEZ - AMAYA, D.B. 1997. Carotenoides y Preparación de Alimentos: La Retención de los Carotenoides Provitamina A en Alimentos Preparados, Procesados y Almacenados. Fac. Engenharia de Alimentos, Universidade Estadual de Campinas. Brasil. $105 p$.

25. ROSTAGNO, H.S. 2005. Tablas brasileñas para aves y cerdos: composición de alimentos y requerimientos nutricionales. 2da. Ed. Universidad Federal de Viçosa. Brasil. 186p.

26. SALINAS, M.Y.; SAAVEDRA, A.S.; SORIA, R.J.; ESPINOSA, T.E. 2008. Características fisicoquímicas y contenido de carotenoides en maíces (Zea mays L.) amarillos cultivados en el estado de México. Agr. Técn. México. 34(3):357-364.
27. SÁNCHEZ, A.; FLORES C., L.B.; LANGLEY, E.; MARÍN, R.; MALDONADO, G.; SÁNCHEZ, S. 1999. Carotenoides: estructura, función, biosíntesis, regulación y aplicaciones. Rev. Latinoam. Microbiol. 41:175-191.

28. SAVÓN, L. 2002. Alimentos altos en fibra para especies monogástricas. Caracterización de la matriz fibrosa y sus efectos en la fisiología digestiva. Rev. Cubana Ciencia Agr. 36(2):91-102.

29. SAELEAW, M.; SCHLEINING, G. 2011. Composition, physicochemical and morphological characterization of pumpkin flour. $11^{\text {th }}$ International Congresson Engineering and Food. Disponible en internet en: http://www.icef11.org/content/papers/fms/FMS328. pdf (con acceso 25/09/2012).

30. SINDIK, M.; TERRAES, J.C.; SANDOVAL, L.; REVIDATTI, F.; FERNANDEZ, R.; BETELLA, A. 2008. Efectos de diferentes relaciones energía/proteína sobre el comportamiento productivo de pollos parrilleros hembras. InVet. 10(1):1-17.

31. TOBAR, D.E.; VALLEJO, F.A.; BAENA, D. 2010. Evaluación de familias de zapallo (Cucurbita moschata Duch.) seleccionadas por mayor contenido de materia seca en el fruto y otras características agronómicas. Acta Agron. 59(1):65-72.

32. UNDERWOOD, E.J.; SUTTLE, N.F. 2003. Los minerales en la nutrición del ganado. 3ra. Ed. Acribia, S.A. Zaragoza, España. 525p.

33. UNITED STATES DEPARTMENT OF AGRICULTURE USDA-. 2008. Agricultural research service. National Nutrient Database for Standard Reference, Release 21.Nutrient Data Laboratory Home Page. Base de datos Acces. Disponible en internet en: http://www. ars.usda.gov/ba/bhnrc/ndl (con acceso 25/09/2012).

34. VACA, A.L. 2003. Producción avícola. 2da reimpresión. EUNED. San José, Costa Rica. 260p.

35. VALDÉS R., M.P.; ORTIZ, G.S.; BAENA, G.D.; VALLEJO C., F.A. 2010. Evaluación de poblaciones de zapallo Cucurbita moschata Duch. Por caracteres de importancia agroindustrial. Acta Agron. 59(1):91-96.

36. VALLEJO, F.A.; ESTRADA, E.I. 2004. Producción de hortalizas de clima cálido. Universidad Nacional de Colombia. Palmira, Colombia. 191p.

37. ZAMAN, Q.U.; MUSHTAQ, T.; NAWAZ, H.; MIRZA, M.A.; MAHMOOD, S. 2008. Effect of varying dietary ener- 
gy and protein on broiler performance in hot climate. Animal Feed Sci. Techn. 146(3-4):302-312.

38. ZANUSSO, J.T.; MIRANDA DE OLIVEIRA, R.F.; LOPES, J.; FERREIRA, R.A.; ROSTAGNO, H.S. 1999. Níveis de energía metabolizável para frangos de Corte de 1 a 21 días de idade mantidos em ambiente de conforto térmico. Rev. Bras. Zootec. 28(5):1068-1074.
Recibido: Abril 6 de 2014

Aceptado: Enero 21 de 2015

Cómo citar:

Ubaque, C.C.; 1, Orozco, L.V.; Ortiz, S.; Valdés, M.P.; Vallejo, F.A. 2015. Sustitución del maíz por harina integral de zapallo en la nutrición de pollos de engorde. Rev. U.D.C.A. Act. \& Div. Cient. 18(1): 137-146. 